Metabolic Consequences of Chronic Renal Failure (2nd Edition)

By Michael R. Wills. Pp. 216, illustrated. HM+M Publishers, Aylesbury, Bucks, 1978. $£ 10.50$.

This took gives a comprehensive account of the metabolic chaos resulting from advanced chronic renal disease. Considering the title, it could be argued that too great a proportion of the text is devoted to normal renal mechanisms; however, since there are so many gaps in our knowledge of the precise metabolic causes of individual clinical manifestations, such consideration of the normal is not out of place. The section devoted to calcium and phosphorus metabolism in renal failure is particularly helpful as is discussion of carbohydrate and lipid disturbance. Due account is also given to the problems consequent upon replacement treatment by dialysis and transplantation.

The text suffers a little from repetition, used for emphasis, but the style is easy to read. Apart from the opening chapter, which is vague and might well have been omitted, this is a valuable account of a complex subject and the extensive review of relevant literature is exceptionally good.

\section{Movements of the Eyes}

By R. H. S. Carpenter. Pp. 420, illustrated. Pion, London, 1977. £13.50.

The author, Roger Carpenter, a lecturer in Physiology at Cambridge University, is to be congratulated on producing a scholarly yet practical book on the neuro-physiology of eye movements. He claims his book was written with the more ambitious medical and physiology student in mind, as well as the neurophysiological researcher, but I suspect this book will have a much wider readership. It certainly deserves it.

Movements of the Eyes is divided into three sections. Part 1 deals with Function describing the various typles of eye movement with experimental evidence and data where applicable. At first sight it might appear that Part 2 , concerning Structure, should be at the beginning but there is no doubt that there is much to commend covering Function first of all. Structure in Part 2 describes the anatomy of the eye muscles and their detailed central nervous pathways. Of particular value in this part are the descriptions of experiments on the mechanical properties of the eyes in movement, so fundamental to the experimenter. To summarize a detailed discussion in this second part of the book there is a good little diagrammatic representation of nerve pathways serving oculomotor function which forms a useful reference and could usefully have appeared also at the commencement of this part on Function.

Part 3 completes the main content of the book and is concerned with 'the system as a whole', in other words to relate the two previous parts of the book on function and structure to the control mechanisms. This third section is well written coping with the complex, and sometimes hypothetical, central control of eye movements in a relaxed readable style. The author attempts a synthesis of eye muscle control in the closing pages of this part but frankly points out the many unknowns.

A useful final Appendix for those not versed in linear systems analysis is a short introduction to the subject which helps in some parts of the text in this book. References are full and listed in alphabetical order of authors mentioned in the text; a convenient and handy ready reference list.

Altogether an excellent book at a reasonable price and should be read by all those concerned with a detailed knowledge of eye movements. It will be of considerable interest to neuro-physiologists and clinicians each able to extract valuable thought and information from this excellent book.
Multiple Choice Questions in Psychiatry

By G. Glew. Pp. 78, soft cover. Butterworths, London, etc., 1978. £2.95.

Anyone who has attempted to construct multiple choice $c$ questions in clinical fields comes to realize that there are very $\vec{\equiv}$ few facts in clinical medicine. Finding five adequate re- -0 sponses is always difficult. Psychiatry presents majoro problems for those constructing such questions. This little $\bar{\sigma}$ book of $300 \mathrm{MCQs}$ covers the fields of neuroanatomy, neurophysiology, biochemistry, neuroendocrinology, action $\frac{}{1}$

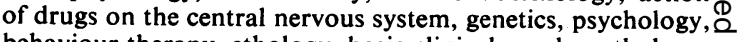
behaviour therapy, ethology, basic clinical psychopathology, statistics and epidemiology.

This reviewer considers that the questions are of high $\overrightarrow{0}$ standard, better and less ambiguous than is sometimes the case in qualifying and postgraduate examinations in medi- $\vec{\omega}$ cine. Even so, occasionally problems of face validity arise $\vec{W}$ with items. The purpose of the book is to familiarize would-D be candidates with such tests. The questions in this book also $\frac{0}{3}$ enable them to learn some psychiatry quite simply and effectively. If you know the answers to these $300 \mathrm{MCQs}$ then you have a very adequate core of knowledge for, say, the MRC Psych. preliminary test.

Whether MCQs which these days are highly regarded as a measure of academic ability are really the best way of $\mathcal{V}$ assessing candidates for professional examinations is another matter. It is to be hoped that as the years pass, they $\sigma$ will be kept in their place as merely part of a global mulpp 음 factorial assessment.

\section{Textbook of Human Genetics (2nd Edition)}

By Max Levitan and Ashley Montagu. Revised Max Levitan. Pp. 1012 , illustrated. Oxford Universig Press, New York, 1978. $£ 15.50$.

In 1975, a report published by the National Academy Science, U.S.A., revealed the need for formal training genetics among medically qualified practitioners (Genetical Report, 1975). The report showed that the majority of doctors had no formal instruction in genetics and lacked knowledge about the frequency of genetic disease in the $\bar{\partial}$ population. Many lacked experience in identifying families with or at risk of genetic disease.

The report concluded that the diffidence of the medical profession was due to its lack of awareness of the importance $\overrightarrow{\vec{O}}$ of genetic defects as a cause of disease, and recommended 3 that instruction of undergraduates in human genetics should $\overrightarrow{2}$ be mandatory. A similar view was put forward by a working party on Genetic Studies of the Academic Board, University of London, and most medical schools now include a course in human genetics in the curriculum.

Of all the text books which have been recommended for such a course, Levitan's Textbook of Human Genetics has proved to be the one most acceptable to the students. General text books of genetics are too wide ranging, whereas specialized books on medical genetics avoid discussing the complex mathematics of population genetics and multiple 윽 gene loci. Levitan has successfully related theoretical and clinical human genetics by presenting principles and citing examples to illustrate them and the production of this second edition is testimony to its popularity with students. N

The second edition is as readable and informative as the $O$ first, with clear print, good index, high quality illustrations $N$ and logical text enlivened by numerous anecdotes and carefully chosen examples. Meiosis, mitosis, the human chromo- $\omega$ some complement, the structure of the gene, Mendelian law and the mechanism of gene action are described in chapters $\theta$ I to VII as before. Chapters VIII to XIX cover methods of $\frac{\mathscr{C}}{\overparen{T}}$ genetic inference in man, the origin and maintenance of $\mathbb{D}$ 